\title{
Prevalence of intestinal parasitic infections and associated risk factors for hookworm infections among primary schoolchildren in rural areas of Nakhon Si Thammarat, southern Thailand
}

Chuchard Punsawad ${ }^{1,2^{*}}$ (D) Nonthapan Phasuk ${ }^{1,2}$, Suchirat Bunratsami ${ }^{1,2}$, Kanjana Thongtup ${ }^{1}$, Parnpen Viriyavejakul ${ }^{3}$, Sarawoot Palipoch ${ }^{1}$, Phanit Koomhin ${ }^{1}$ and Somchok Nongnaul ${ }^{4}$

\begin{abstract}
Background: Soil-transmitted helminth (STH) infections are among the most common type of infections worldwide and are widely distributed in tropical areas. In rural areas of southern Thailand where most land is used for agriculture, children are at risk of acquiring parasites, especially STHs. Assessing the current situation regarding parasitic infection in these areas is a prerequisite for developing appropriate control measures. This study is aimed at determining the prevalence of intestinal parasitic infections, the intensity of STH infections and the associated risk factors among primary schoolchildren in Nopphitam District, Nakhon Si Thammarat Province, Thailand.

Methods: A cross-sectional study involving 299 schoolchildren between 7 and 12 years of age was conducted between January and March 2016. A questionnaire administered by direct interviews was used to collect sociodemographic information and data on associated risk factors. Stool samples were processed using direct wet smears, formalin-ethyl acetate sedimentation concentration, and the modified Kato-Katz technique.

Results: The overall prevalence of intestinal parasites among the 299 children was 16\% (48 of 299), with 32 children infected with hookworms (10.7\%), 10 with Blastocystis hominis (3.3\%), seven with Giardia intestinalis (1.6\%), one with Enterobius vermicularis (0.3\%), and one with Trichuris trichiura (0.3\%). The hookworm infection intensity, measured by the median eggs per gram (EPG) of stool, was 1200 EPG (Interquartile range (IQR): 360-3200). Most children had light-intensity hookworm infections, but two had heavy-intensity infections. When participants included in the sample were classified by age, children 10-12 years old demonstrated higher intestinal parasite prevalence than those aged 7-9 years (adjusted odds ratio $(\mathrm{AOR})=2.3,95 \% \mathrm{Cl}: 1.1-4.9, P=0.030$ ). Inadequate handwashing before meals was statistically associated with hookworm infections ( $\mathrm{AOR}=2.3 ; 95 \% \mathrm{Cl}: 1.1-4.8, P=0.037$ ).

Conclusions: This study highlights that hookworms are the most prevalent STH infection in the study area. Older age group (10-12 years) and inadequate handwashing before meals were statistically associated with hookworm infections. Accordingly, appropriate strategies and education on personal and environmental hygiene should be implemented. Moreover, the cost-effectiveness of mass drug administration in this area should be further investigated.
\end{abstract}

Keywords: Hookworms, Intestinal parasites, Soil-transmitted helminth, Schoolchildren, Southern Thailand

\footnotetext{
* Correspondence: chuchard.pu@wu.ac.th

${ }^{1}$ School of Medicine, Walailak University, Nakhon Si Thammarat, Thailand

${ }^{2}$ Tropical Diseases and Parasitic Infectious Diseases Research Group, Walailak

University, Nakhon Si Thammarat, Thailand

Full list of author information is available at the end of the article
}

(c) The Author(s). 2018 Open Access This article is distributed under the terms of the Creative Commons Attribution 4.0 International License (http://creativecommons.org/licenses/by/4.0/), which permits unrestricted use, distribution, and reproduction in any medium, provided you give appropriate credit to the original author(s) and the source, provide a link to the Creative Commons license, and indicate if changes were made. The Creative Commons Public Domain Dedication waiver (http://creativecommons.org/publicdomain/zero/1.0/) applies to the data made available in this article, unless otherwise stated. 


\section{Background}

Soil-transmitted helminths (STHs) are intestinal worms that infect humans and are transmitted through contaminated soil. STH infection is considered a neglected tropical disease [1]. The main species that infect humans are roundworms (Ascaris lumbricoides), whipworms (Trichuris trichiura), and hookworms (Necator americanus and Ancylostoma duodenale) [2-4]. Approximately 2 billion people are infected with STHs worldwide [5]. The World Health Organization (WHO) has stated that school-aged children are particularly at risk for STH infection [5]. STH infection in children can lead to impaired physical, intellectual, and cognitive development $[2,6,7]$. Several risk factors are associated with STH transmission and infection, namely, age, poor environmental sanitation, poor personal hygiene, geography, socioeconomic status, and occupation $[2,3]$.

Several studies have reported the STH infection prevalence in schoolchildren in Thailand at percentages ranging from 5.6 to $75.1 \%$ [8-13]. The predominant STH infection type varies by geographical region. For decades in southern Thailand, the most prevalent parasite infection among all age groups was hookworms [14, 15]. During the 1980s, hookworm infection prevalence in southern Thailand reached $76 \%$. At that time, treatment with a single dose of mebendazole was implemented through a national program [16]. Twenty years later, in 2002, along with better knowledge of personal health and hygiene and more accessible health services, the hookworm infection prevalence among children decreased to $21.1 \%$ [17]. Currently, due to rapid urbanization in Thailand, parasitic infections are thought to be further decreasing and have been given relatively little attention by the national government.

Although several studies have been conducted on the distribution and prevalence of intestinal parasitic infections in southern Thailand, epidemiological information on STHs and other intestinal parasitic infections is lacking for several remote areas. Therefore, this study is aimed at assessing the prevalence of STH and other intestinal parasitic infections among schoolchildren in Nopphitam District and identifying potential risk factors for infection or transmission in the study area.

\section{Methods}

\section{Study design and setting}

A cross-sectional study was conducted from January to March 2016 at twelve primary schools in Nopphitam District, Nakhon Si Thammarat Province in southern Thailand. Nopphitam District is located approximately $780 \mathrm{~km}$ south of the Thai capital of Bangkok and $80 \mathrm{~km}$ from the city of Nakhon Si Thammarat. The average temperature is $27.1{ }^{\circ} \mathrm{C}$, with a low of $25.8{ }^{\circ} \mathrm{C}$ in January and a high of $29.3{ }^{\circ} \mathrm{C}$ in May. Annual rainfall is $1454.3 \mathrm{~mm}$ (Climatological Center, Thai Meteorological Department, Annual Report 2015). The Thailand
Department of Provincial Administration estimates that the total population of these subdistricts was 33,183 in 2015. Although Nakhon Si Thammarat Province has been rapidly urbanized in the past 10 years, Nopphitam District has remained rural with only one local public hospital and twelve primary schools. The main access road is non-asphalt, while the other roads are asphalt. Most land is used for farming and rubber plantations.

\section{Study population and sample size}

The study population consisted of children from 7 to 12 years of age attending elementary schools in Nopphitam District. The sample size was determined using the single proportion population formula:

$$
\mathrm{Z}^{2} \mathrm{p}(1-\mathrm{p}) / \mathrm{d}^{2}
$$

where $\mathrm{p}=$ prevalence of intestinal parasites from a previous study, $\mathrm{d}=$ margin of error, and $\mathrm{Z}=$ the value from a standard normal distribution corresponding to the desired confidence level, which equals 1.96 for a $95 \%$ CI. This formula was calculated based on a prevalence rate of $24.1 \%$ from a previous study [17], with a margin of error of 0.05 and a confidence level of $95 \%$. The appropriate sample size was determined to be 286 . We assumed that the final sample size would be reduced by $5 \%$ due to incomplete data and thus aimed for a sample size of 300. Students who had been treated for intestinal helminthiasis within 1 month of the collection date, declined participation, or could not pass stool on the collection date were excluded from the study. Finally, using class rosters from twelve primary schools as the sample frame, the sample was selected using a systematic random sampling technique.

\section{Questionnaire survey}

A structured questionnaire (Additional file 1) was developed and used to collect demographic data (i.e., age, gender, and education level) and information on possible risk factors (i.e., handwashing before meals, eating raw meat, etc.). The questionnaire was administered by two trained interviewers who directly interviewed the participating schoolchildren.

\section{Stool sample collection and laboratory procedure}

All children were given oral instructions for handling and collecting fecal samples. Each student received a clean plastic container on the day before specimen collection. A single fresh stool sample of approximately 2-10 g was collected from each student. The students returned their stool specimens in labeled containers. Each specimen was checked for correct labeling, quantity, and collection procedure and transported immediately to the parasitology laboratory of the School of Medicine, Walailak University. Part of each stool specimen was processed immediately 
using a direct microscopic technique with saline and iodine solution to detect protozoan trophozoites, cysts, oocysts, and helminth eggs. The remainder of each stool specimen was analyzed by formalin-ethyl acetate sedimentation concentration, which increases the chances of detecting parasitic organisms in small numbers [18]. Briefly, $1 \mathrm{~g}$ of stool sample was suspended in $5 \mathrm{ml}$ of $10 \%$ formalin, sieved with cotton gauze, and transferred to a $15-\mathrm{ml}$ conical tube, which was then centrifuged for $10 \mathrm{~min}$ at $500 \times$ g. Following this, $8 \mathrm{ml}$ of $10 \%$ formalin and $4 \mathrm{ml}$ of ethyl acetate were added to the sediment, which was shaken vigorously in an inverted position for $30 \mathrm{~s}$ and centrifuged for $10 \mathrm{~min}$ at $500 \times \mathrm{g}$. Subsequently, the supernatant was discarded, and the remaining sediment was observed under light microscopy at $10 \times$ and $40 \times$ objectives to detect the eggs and larvae of helminths and cysts and the trophozoites of protozoan parasites. The parasites were morphologically diagnosed under the Centers for Disease Control and Prevention diagnostic reference [18]. Iodine solution was used to detect and identify cysts of protozoan parasites. The modified Kato-Katz technique was also performed to determine the STH infection intensity [19]. Eggs were quantified by species and calculated as eggs per gram (EPG) of stool. According to WHO recommendations, the hookworm infection intensities were classified as light-intensity (1-1999 EPG), moderate-intensity (2000-3999 EPG), and heavy-intensity ( $\geq 4000$ EPG), respectively. [20]. To eliminate observer bias, each stool sample was examined by two trained senior medical laboratory technologists who were uninformed of the study participants' identification and behavioral risk factors.

\section{Data analysis}

Data were entered, cleaned, and analyzed using IBM SPSS Statistics for Windows, Version 23.0. Armonk, NY: IBM Corp. Median \& interquartile ranges (IQR) for continuous variables and proportions for categorical variables were computed. A mixed effect logistic regression analysis was used to assess the association of independent variables with hookworm infections. The hierarchical structure of the model was the twelve different schools, which were used as random effects in order to account for clustering. Crude odds ratio (COR) of the binary outcome variable (with and without hookworm infection) for each independent variable was assessed by univariable analysis. All variables with $P$-values less than 0.05 in the univariable analysis were analyzed using multivariable analysis to adjust for possible confounders. The final model was interpreted by the adjusted odds ratio (AOR) with a 95\% confidence interval (CI). Differences were considered statistically significant when the $P$-value was less than 0.05 .

\section{Results}

Sociodemographic characteristics

A total of 299 participants between 7 and 12 years of age comprised the study sample. The median age was 9 years (IQR: $8-11$ ), with $53.8 \%$ of participants (161/299) less than 10 years of age. Males represented 57.5\% (172/299) of enrolled participants (Table 1).

\section{Prevalence of intestinal parasitic infections}

Of the 299 stool specimens examined, the overall parasitic infection prevalence was $16 \%(48 / 299)$. Hookworms were the predominant parasites, with 32 infection cases (10.7\%). The prevalence of intestinal parasitic and protozoal infections is shown in Fig. 1. While most positive cases were single infections, four children (1.3\%) had double infections: two had G. intestinalis and hookworm infections; one had B. hominis and hookworm infections, and one had T. trichiura and hookworm infections. The median EPGs of the hookworms and T. trichiura were 4550 (IQR: 40-32,360) and 92, respectively. Most hookworm infections (30 of 32) were light-intensity; however, two children had heavy-intensity infections.

\section{Associations of independent variables with hookworm infections}

The study results indicated that hookworm was the most prevalent infection; therefore, we assessed the association between hookworm infections and several potential predictor variables to determine risk factors for the infection. When classified by age, the prevalence of intestinal parasitic infections in children aged 10-12 years was higher (15.2\%) than for those aged $7-9$ years $(6.8 \%)(\mathrm{COR}=2.2$,

Table 1 Sociodemographic characteristics of primary schoolchildren in Nopphitam District, Nakhon Si Thammarat Province, Thailand, 2016

\begin{tabular}{|c|c|c|}
\hline Characteristics & Number & Percentage \\
\hline \multicolumn{3}{|l|}{ Gender } \\
\hline Male & 172 & 57.5 \\
\hline Female & 127 & 42.5 \\
\hline \multicolumn{3}{|l|}{ Age group (years) } \\
\hline $7-9$ & 161 & 53.8 \\
\hline $10-12$ & 138 & 46.2 \\
\hline \multicolumn{3}{|l|}{ Level of education (grade) } \\
\hline 1 & 36 & 12.0 \\
\hline 2 & 59 & 19.7 \\
\hline 3 & 66 & 22.1 \\
\hline 4 & 64 & 21.4 \\
\hline 5 & 23 & 7.7 \\
\hline 6 & 51 & 17.1 \\
\hline Buddhism & 299 & 100.0 \\
\hline
\end{tabular}




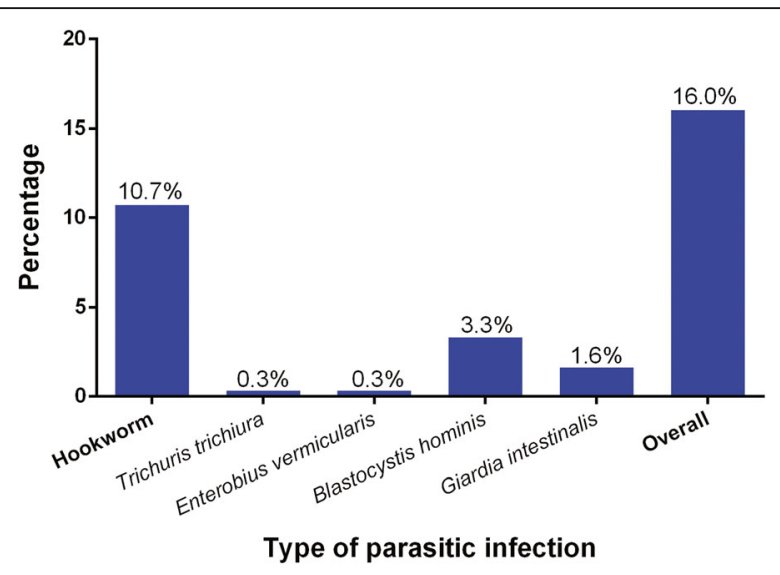

Fig. 1 Prevalence of parasitic infections among primary schoolchildren in Nakhon Si Thammarat, southern Thailand, 2016

95\% CI: $1.1-4.7, P=0.037)$. Children who did not regularly wash their hands before meals were more likely to be infected with hookworms than those who did wash $(\mathrm{COR}=2.2$; 95\% CI: $1.0-4.6, P=0.046)$. In addition, all 32 children with hookworm infections reported eating fresh vegetables. Furthermore, children who practiced open defecation and played on the ground were 1.6 and 1.8 times more likely, respectively, to have hookworm infections than children without these risk factors. However, these associations were not statistically significant $(\mathrm{COR}=1.6 ; 95 \% \mathrm{CI}: 0.5-5.2, \quad P=0.398$ and $\mathrm{COR}=1.8 ; 95 \%$ CI: $0.3-12.5, P=0.539$, respectively) (Table 2).

Multivariable analysis also indicated that age and handwashing before meals were significantly associated with hookworm infections. Children aged 10-12 years were more likely to have hookworm infections than those aged 7-9 years $(\mathrm{AOR}=2.3,95 \% \mathrm{CI}: 1.1-4.9, P=0.030)$. Children who did not regularly wash their hands before meals were more likely to be infected with STH than children who did practice good hand washing $(\mathrm{AOR}=2.3$; 95\% CI: 1.1-4.8, $P=0.037$ ) (Table 2).

\section{Discussion}

We determined the overall prevalence of intestinal parasitic infections among primary school children 7-12 years of age from Nopphitam District, Nakhon Si Thammarat Province in southern Thailand. Of 299 schoolchildren, 48 $(16 \%)$ were positive for at least one intestinal parasite, including both helminths and protozoa. The observed prevalence of intestinal parasites was higher than the prevalences found in similar reports conducted in other parts of Thailand over the past two decades. Those prevalence rates included $4.24 \%$ in central area of Thailand, including Ang Thong, Ayutthaya, and Suphanburi Provinces in 2004 [21]; 13.9\% in Pathum Thani Province in 2010 [10] and $5.4 \%$ in Phitsanulok Province, in lower northern area of Thailand during 2009 and 2010 [22]. The present study found that intestinal parasitic infection remains a public health problem in southern Thailand compared with other parts of the country. Furthermore, it also indicates that helminths and protozoa are prevalent in these areas. In contrast, the prevalence of overall intestinal parasitic infections observed in this study was lower than in previous studies conducted in southern Thailand using the same diagnostic method (formalin-ethyl acetate sedimentation concentration) used in this study, specifically $75.1 \%$ in Narathiwat Province in 2003 [9] and 19.8\% in a national survey conducted in southern Thailand in 2009 [15]. Improved environmental and socioeconomic factors may account for the decreased prevalence of intestinal parasites in these areas. These data show that over two decades, the prevalence of intestinal parasitic infections in southern Thailand has decreased but remains a problem.

Hookworms were the most common helminths found in the schoolchildren, which is consistent with previous reports conducted in southern Thailand [15-17]. However, the observed hookworm infection prevalence of $10.7 \%$ was lower than reports from a similar study in southern Thailand, which reported a prevalence of $21.1 \%$ in 2002 [17], and a national survey, which reported a prevalence of $15.8 \%$ in 2009 [15]. The lower hookworm infection prevalence in the present study may be due to differences in urbanization, improved knowledge of personal hygiene, and improved environmental sanitation, for example, clean and safe water supply, and effective human and animal waste disposal. However, further work is needed to detect the health impacts of hookworm infections on children in the studied community.

From the 32 schoolchildren with STH infections, one was coinfected with hookworms and T. trichiura. Ascaris lumbricoides, another STH, was not found among these schoolchildren. This low T. trichiura infection prevalence and the absence of $A$. lumbricoides are consistent with a previous report by Anantaphruti et al., 2002, which reported a hookworm prevalence of $21.1 \%$ and a T. trichiura prevalence of 5.4\%, with no A. lumbricoides [17]. The important factors affecting the prevalence of STH in southern Thailand are tropical climatic conditions and moist soil that is suitable for the survival of parasitic eggs. In this study, more than half of study subjects reported risk behaviors related to STH infections, including walking barefoot (54.2\%) and playing on the ground (94\%). These data suggest that health education on personal hygiene should be part of the educational curriculum in primary schools in the study area.

The previous control measures for STH infections in the study area were limited to one school, which is a representative of Nopphitam District where the Ministry of Public Health administers regular fecal examinations every 3 years. Testing results of this program revealed less than a $5 \%$ prevalence of parasitic infections. Accordingly, Nopphitam 
Table 2 Factors associated with hookworm infection among primary schoolchildren in Nopphitam District, Nakhon Si Thammarat Province, Thailand, 2016

\begin{tabular}{|c|c|c|c|c|c|c|c|c|c|}
\hline Variables & Number (\%) & No. positive (\%) & No. negative (\%) & $\mathrm{COR}^{\mathrm{a}}$ & $95 \%^{\mathrm{c}} \mathrm{Cl}$ & $P$-value & $\mathrm{AOR}^{\mathrm{b}}$ & $95 \%{ }^{\mathrm{c}} \mathrm{Cl}$ & $P$-value \\
\hline \multicolumn{10}{|l|}{ Age (years) } \\
\hline $7-9$ & $161(53.8)$ & $11(6.8)$ & $150(93.2)$ & & & & & & \\
\hline $10-12$ & $138(46.2)$ & $21(15.2)$ & $117(84.8)$ & 2.2 & $1.1-4.7$ & $0.037^{*}$ & 2.3 & $1.1-4.9$ & $0.030^{*}$ \\
\hline \multicolumn{10}{|l|}{ Sex } \\
\hline Male & $172(57.5)$ & $20(11.6)$ & $152(88.4)$ & & & & & & \\
\hline Female & $127(42.5)$ & $12(9.4)$ & 115 (90.6) & 1.2 & $0.6-2.6$ & 0.580 & & & \\
\hline \multicolumn{10}{|c|}{ Handwashing before meals } \\
\hline Yes & $224(74.9)$ & $19(8.5)$ & $205(91.5)$ & & & & & & \\
\hline No & $75(25.1)$ & $13(17.3)$ & $62(82.7)$ & 2.2 & $1.0-4.6$ & $0.046^{*}$ & 2.3 & $1.1-4.8$ & $0.037^{*}$ \\
\hline \multicolumn{10}{|c|}{ Drinking water source } \\
\hline Bottled water & $94(31.4)$ & $8(8.5)$ & $86(91.5)$ & & & & & & \\
\hline Tap water & $106(35.5)$ & $19(17.9)$ & $87(82.1)$ & 2.2 & $0.9-5.4$ & 0.069 & & & \\
\hline Rain & $36(12.0)$ & $1(2.8)$ & $35(97.2)$ & 0.5 & $0.1-2.7$ & 0.408 & & & \\
\hline Water filter & $63(21.1)$ & $4(6.3)$ & $59(93.7)$ & 0.8 & $0.2-2.6$ & 0.685 & & & \\
\hline \multicolumn{10}{|l|}{ Eating raw meat } \\
\hline No & $110(36.8)$ & $10(9.1)$ & $100(90.9)$ & & & & & & \\
\hline Yes & $189(63.2)$ & $22(11.6)$ & $167(88.4)$ & 1.4 & $0.6-3.1$ & 0.453 & & & \\
\hline \multicolumn{10}{|c|}{ Eating fresh vegetables } \\
\hline No & $\begin{array}{l}17 \\
(5.7)\end{array}$ & $0(0)$ & $17(100.0)$ & & & & & & \\
\hline Yes & $282(94.3)$ & $32(11.3)$ & $250(88.7)$ & 4.4 & $0.2-77.9$ & 0.314 & & & \\
\hline \multicolumn{10}{|l|}{ Open defecation } \\
\hline No & $272(91.0)$ & $28(10.3)$ & $244(89.7)$ & & & & & & \\
\hline Yes & $27(9.0)$ & $4(14.8)$ & $23(85.2)$ & 1.6 & $0.5-5.2$ & 0.398 & & & \\
\hline \multicolumn{10}{|c|}{ Contact with domestic animals } \\
\hline No & $24(8.0)$ & $4(16.7)$ & $20(83.3)$ & & & & & & \\
\hline Yes & $275(92.0)$ & $28(10.2)$ & $247(89.8)$ & 0.5 & $0.2-1.7$ & 0.297 & & & \\
\hline \multicolumn{10}{|c|}{ Wearing shoes outside the house } \\
\hline Yes & $137(45.8)$ & $17(12.4)$ & $120(87.6)$ & & & & & & \\
\hline No & $162(54.2)$ & $15(9.3)$ & $147(90.7)$ & 0.7 & $0.4-1.5$ & 0.419 & & & \\
\hline \multicolumn{10}{|c|}{ Playing on ground } \\
\hline No & $18(6.0)$ & $1(5.6)$ & $17(94.4)$ & & & & & & \\
\hline Yes & $281(94.0)$ & $31(11.0)$ & $250(89.0)$ & 1.8 & $0.3-12.5$ & 0.539 & & & \\
\hline
\end{tabular}

*Significant association

a COR: crude odds ratio by univariable analysis

${ }^{b}$ AOR: adjusted odds ratio by multivariable analysis

${ }^{c} \mathrm{Cl}$ : $95 \%$ confidence interval

District is considered a low-prevalence area by the Ministry of Public Health, and drug treatment is administered on a three-year basis for students in the school. The results of this study reveal that control measures are not universally effective in the study area, as the prevalence of hookworm infections remained as high as $10.7 \%$. Although WHO recommends that mass drug administration (MDA) should be performed once annually when the baseline STH infection prevalence in a community is over $20 \%$ [5], we suggest that
MDA programs should be considered in the study area even though prevalence did not reach $20 \%$, as this practice is a mainstay of controlling STH infections.

As this study revealed that hookworm was the most common parasitic infection among schoolchildren in the studied community, multivariable analysis identified age and handwashing before meals as statistically significant associated risk factors for hookworm infection. Schoolchildren in the 10- to 12-year-old group had a significantly 
higher percentage of hookworm infections than those in the 7- to 9-year-old group. This trend was also observed in studies conducted in other countries [21-26]. This may be explained because as children grow older, their outdoor activities increase, thus increasing their STH exposure.

Moreover, schoolchildren who did not regularly practice handwashing before meals were significantly infected with hookworms at a rate 2.3 times higher than those who did wash. Although these findings were not statistically significant, children who practiced open defecation and children who played on the ground had 1.6 and 1.8 times higher risks, respectively, of having hookworm infections than their counterparts. This may be explained because the hookworm's mode of transmission is not limited to skin penetration but may also occur by the fecal-oral route; thus, handwashing before meals played an important role in acquiring hookworms in our study.

For protozoal infections, the most predominant protozoa were B. hominis, with 10 infections (3.3\%), and G. intestinalis, with 7 infections (2.3\%). B. hominis has been traditionally regarded as a harmless parasite of humans. However, it can cause symptoms such as abdominal pain, constipation, diarrhea, and irritable bowel syndrome [27, 28]. Five or more $B$. hominis cells per $40 \times$ magnification field seen during direct microscopic examination of a stool smear wet mount is considered to cause clinical illness [29]. In this study, all fecal samples with $B$. hominis revealed more than five cells per $40 \times$ magnification, hence, this protozoa might cause gastrointestinal symptoms among these children, however, we obtained no information on clinical details. These $B$. hominis infections could result from ingestion of contaminated water or food. Additional study on this protozoal burden is needed. Further, more sensitive tests, such as molecular techniques, should be considered for identifying these protozoa [18]; however, such tests are generally expensive and require skilled technicians.

This study had the following limitations. Each method used in our study has varying sensitivity among STHs. The sensitivities are highest for modified Kato-Katz technique with $63.8 \%, 82.2 \%$ and $59.5 \%$ for $A$. lumbricoides, $T$. trichiura, and hookworm, respectively [30]. Secondly, formalin-ethyl acetate sedimentation concentration technique has sensitivities of $56.9 \%, 81.2 \%$ and $53.0 \%$ for $A$. lumbricoides, T. trichiura, and hookworm, respectively [30]. The lowest sensitivity was noted for the direct microscopy method with $52.1 \%, 62.8 \%$ and $42.8 \%$ for $A$. lumbricoides, T. trichiura, and hookworm, respectively [30]. Obtaining multiple stool samples is recommended to improve diagnostic sensitivity, especially for hookworm infection [31], hence, using a single-day fecal examination to determine helminth presence was the major limitation of our study. Furthermore, associated risk factor items were restricted to questionnaire data and might not entirely represent all associated factors. Finally, this study was conducted in Nopphitam District located in southern Thailand, which has distinct tropical hot and humid climate compared with other parts of the country, so the results might not be applied to other geographical settings, especially those outside southern Thailand. The strengths of this study include that the parasite detection yield was increased by using combination of three methods, namely, direct wet smears, the formalin-ethyl acetate concentration technique and a modified Kato-Katz technique [32, 33]. Furthermore, these tests yield high specificity ranged from 97.5 to $99.6 \%$ [30]. In addition, this study is the first to report parasitic infection prevalence among children in Thailand in the past 7 years.

\section{Conclusions}

Hookworm remains the most predominant STH infection among schoolchildren in rural areas of Nakhon $\mathrm{Si}$ Thammarat. Older age group (10-12 years) and inadequate handwashing before meals were statistically associated with hookworm infections. Due to the substantial intestinal parasitic infection prevalence (especially of hookworms), risk factor-related behaviors among schoolchildren, and suitable humidity for STHs in southern Thailand, appropriate control measures should be taken to reduce the prevalence of STH infections in this community, including education on personal hygiene and environmental sanitation and the development of awareness strategies and MDA programs.

\section{Additional file}

Additional files 1: Questionnaire on demographic data and possible risk factors. (PDF $481 \mathrm{~kb}$ )

\section{Abbreviations}

STH: Soil-transmitted helminth; EPG: eggs per gram; AOR: adjusted odds ratio; Cl: confidence interval; IQR: interquartile range; COR: crude odds ratio; MDA: mass drug administration; WHO: World Health Organization

\section{Acknowledgements}

The authors wish to thank the schoolchildren from Nopphitam District who participated in the study. The authors would like to acknowledge the directors and teachers of all primary schools in Nopphitam District, Nakhon Si Thammarat Province, for their support throughout this study. Acknowledgments also go to Miss Potiga Chotipong for preparing the reagents and laboratory instruments.

\section{Funding}

This study was funded by the Institute of Research and Development, Walailak University (WU59107). The funder had no role in study design, data collection and analysis, decision to publish, or preparation of the manuscript.

Availability of data and materials

All data generated or analyzed during this study are included in this published article (and its Additional file). 
and SB analyzed the data. CP, NP and PV wrote the manuscript. All authors read and approved the final manuscript.

\section{Ethics approval and consent to participate}

This study was approved by the Ethics Committee on Human Rights Related to Research Involving Human Subjects, Walailak University, prior to recruiting participants (Protocol no. 15/050). Written informed consent was obtained from the children's parents or legal guardians, after clearly explaining the research objectives.

\section{Consent for publication}

Not applicable.

\section{Competing interests}

The authors declare that they have no competing interests.

\section{Publisher's Note}

Springer Nature remains neutral with regard to jurisdictional claims in published maps and institutional affiliations.

\section{Author details}

'School of Medicine, Walailak University, Nakhon Si Thammarat, Thailand. ${ }^{2}$ Tropical Diseases and Parasitic Infectious Diseases Research Group, Walailak University, Nakhon Si Thammarat, Thailand. ${ }^{3}$ Department of Tropical Pathology, Faculty of Tropical Medicine, Mahidol University, Bangkok, Thailand. ${ }^{4}$ Nopphitam Hospital, Nakhon Si Thammarat, Thailand.

\section{Received: 4 March 2018 Accepted: 7 September 2018}

\section{Published online: 14 September 2018}

\section{References}

1. World Health Statistics. 2013. http://www.who.int/gho/publications/world_ health_statistics/EN_WHS2013 Full.pdf. Accessed 1 Mar 2018.

2. Bethony J, Brooker S, Albonico M, Geiger SM, Loukas A, Diemert D, Hotez PJ. Soil-transmitted helminth infections: ascariasis, trichuriasis, and hookworm. Lancet. 2006;367(9521):1521-32.

3. de Silva NR, Brooker S, Hotez PJ, Montresor A, Engels D, Savioli L. Soiltransmitted helminth infections: updating the global picture. Trends Parasitol. 2003;19(12):547-51.

4. Soil-transmitted Helminths [https://www.cdc.gov/parasites/sth/]. Accessed 1 Mar 2018.

5. Soil-transmitted helminth infections. http://www.who.int/mediacentre/ factsheets/fs366/en/. Accessed 1 Mar 2018.

6. Oliveira D, Ferreira FS, Atouguia J, Fortes F, Guerra A, Centeno-Lima S Infection by intestinal parasites, stunting and Anemia in school-aged children from southern Angola. PLoS One. 2015;10(9):e0137327.

7. Rajoo Y, Ambu S, Lim YA, Rajoo K, Tey SC, Lu CW, Ngui R. Neglected intestinal parasites, malnutrition and associated key factors: a population based cross-sectional study among indigenous communities in Sarawak, Malaysia. PLoS One. 2017;12(1):e0170174.

8. Boonjaraspinyo S, Boonmars T, Kaewsamut B, Ekobol N, Laummaunwai P, Aukkanimart R, Wonkchalee N, Juasook A, Sriraj P. A cross-sectional study on intestinal parasitic infections in rural communities, Northeast Thailand. Korean J Parasitol. 2013;51(6):727-34.

9. Jiraamonninit C, Wongkamchai S, Santabutr W, Loymek S, Monkong N Nochot H, Wankhom S, Choochote W. The prevalence of intestinal parasitic infections among schoolchildren with annual anthelminthic treatment in Narathiwat province, Thailand. J Trop Med Parasitol. 2006;29(2):45-50.

10. Kitvatanachai $S$, Rhongbutsri P. Intestinal parasitic infections in suburban government schools, Lak Hok subdistrict, Muang Pathum Thani, Thailand. Asian Pac J Trop Med. 2013;6(9):699-702.

11. Wongstitwilairoong B, Srijan A, Serichantalergs O, Fukuda CD, McDaniel P, Bodhidatta L, Mason CJ. Intestinal parasitic infections among pre-school children in Sangkhlaburi, Thailand. Am J Trop Med Hyg. 2007;76(2):345-50.

12. Waikagul J, Krudsood S, Radomyos P, Radomyos B, Chalemrut K, Jonsuksuntigul P, Kojima S, Looareesuwan S, Thaineau W. A cross-sectional study of intestinal parasitic infections among schoolchildren in Nan Province, northern Thailand. Southeast Asian J Trop Med Public Health. 2002;33(2):218-23.
13. Warunee N, Choomanee L, Sataporn P, Rapeeporn Y, Nuttapong W, Sompong S, Thongdee S, Bang-On S, Rachada K. Intestinal parasitic infections among school children in Thailand. Trop Biomed. 2007;24(2):83-8.

14. Jongsuksuntigul P, Manatrakul D, Wongsaroj T, Krisanamara K, Sawatdimongkol S, Wongsaroj S. Evaluation of helminthiasis control program in Thailand at the end of the 8th health development plan, 2001. J Trop Med Parasitol. 2003; 26(1):38-46.

15. Wongsaroj T, Nithikathkul C, Rojkitikul W, Nakaia W, Royal L, Rammasut P. National survey of helminthiasis in Thailand. Asian Biomed. 2014;8(6):779-83.

16. Chongsuvivatwong $V$, Pas-Ong S, Ngoathammatasna W, McNeil D, Vithsupakorn K, Bridhikitti V, Jongsuksuntigul P, Jeradit C. Evaluation of hookworm control program in southern Thailand. Southeast Asian J Trop Med Public Health. 1994;25(4):745-51.

17. Anantaphruti MT, Jongsuksuntigul P, Imsomboon T, Nagai N, Muennoo C, Saguankiat S, Pubampen S, Kojima S. School-based helminthiases control: I. A baseline study of soil-transmitted helminthiases in Nakhon Si Thammarat Province, Thailand. Southeast Asian J Trop Med Public Health. 2002;33(Suppl 3):113-9.

18. DPDx - Laboratory identification of parasitic diseases of public health concern. https://www.cdc.gov/dpdx/diagnosticprocedures/stool/index.html. Accessed 1 Mar 2018.

19. Katz N, Chaves A, Pellegrino J. A simple device for quantitative stool thicksmear technique in Schistosomiasis mansoni. Rev Inst Med Trop Sao Paulo. 1972;14(6):397-400.

20. Helminth control in school-age children. A guide for managers of control programmes. http://apps.who.int/iris/bitstream/10665/44671/1/ 9789241548267_eng.pdf. Accessed 1 Mar 2018.

21. Shiferaw MB, Mengistu AD. Helminthiasis: hookworm infection remains a public health problem in Dera District, South Gondar, Ethiopia. PLoS One. 2015;10(12):e0144588.

22. Wegayehu T, Tsalla T, Seifu B, Teklu T. Prevalence of intestinal parasitic infections among highland and lowland dwellers in Gamo area, South Ethiopia. BMC Public Health. 2013;13:151.

23. Gelaw A, Anagaw B, Nigussie B, Silesh B, Yirga A, Alem M, Endris M, Gelaw $B$. Prevalence of intestinal parasitic infections and risk factors among schoolchildren at the University of Gondar Community School, Northwest Ethiopia: a cross-sectional study. BMC Public Health. 2013;13:304.

24. Anantaphruti MT, Maipanich W, Muennoo C, Pubampen S, Sanguankiat S. Hookworm infections of schoolchildren in southern Thailand. Southeast Asian J Trop Med Public Health. 2002;33(3):468-73.

25. Moore CE, Nget P, Saroeun M, Kuong S, Chanthou S, Kumar V, Bousfield R, Nader J, Bailey JW, Beeching NJ, et al. Intestinal parasite infections in symptomatic children attending hospital in Siem Reap, Cambodia. PLoS One. 2015;10(5):e0123719.

26. Shumbej T, Belay T, Mekonnen Z, Tefera T, Zemene E. Soil-transmitted helminths and associated factors among pre-school children in Butajira town, south-Central Ethiopia: a community-based cross-sectional study. PLoS One. 2015:10(8):e0136342.

27. Qadri SM, al-Okaili GA, al-Dayel F. Clinical significance of Blastocystis hominis. J Clin Microbiol. 1989;27(11):2407-9.

28. Yakoob J, Jafri W, Jafri N, Khan R, Islam M, Beg MA, Zaman V. Irritable bowel syndrome: in search of an etiology: role of Blastocystis hominis. Am J Trop Med Hyg. 2004;70(4):383-5.

29. Sheehan DJ, Raucher BG, McKitrick JC. Association of Blastocystis hominis with signs and symptoms of human disease. J Clin Microbiol. 1986;24(4):548-50.

30. Nikolay B, Brooker SJ, Pullan RL. Sensitivity of diagnostic tests for human soil-transmitted helminth infections: a meta-analysis in the absence of a true gold standard. Int J Parasitol. 2014;44(11):765-74.

31. Sayasone S, Utzinger J, Akkhavong $K$, Odermatt P. Repeated stool sampling and use of multiple techniques enhance the sensitivity of helminth diagnosis: a cross-sectional survey in southern Lao People's Democratic Republic. Acta Trop. 2015;141(Pt B):315-21.

32. Knopp S, Mgeni AF, Khamis IS, Steinmann P, Stothard JR, Rollinson D, Marti $\mathrm{H}$, Utzinger J. Diagnosis of soil-transmitted helminths in the era of preventive chemotherapy: effect of multiple stool sampling and use of different diagnostic techniques. PLoS Negl Trop Dis. 2008;2(11):e331.

33. Steinmann P, Du ZW, Wang LB, Wang XZ, Jiang JY, Li LH, Marti H, Zhou XN, Utzinger J. Extensive multiparasitism in a village of Yunnan province, People's Republic of China, revealed by a suite of diagnostic methods. Am J Trop Med Hyg. 2008;78(5):760-9. 